\title{
Five Years after Discovery Abnormal Neutrino Radioactive-Isotope (ANRI) Absorption
}

\author{
O. B. Khavroshkin, V. V. Tsyplakov \\ Institute of Physics of the Earth, RAS, Moscow, Russia \\ Email:khavole@ifz.ru
}

How to cite this paper: Khavroshkin, O.B. nad Tsyplakov, V.V. (2018) Five Years after Discovery Abnormal Neutrino RadioactiveIsotope (ANRI) Absorption. Open Access Library Journal, 5: e4869.

https://doi.org/10.4236/oalib.1104869

Received: August 28, 2018

Accepted: October 26, 2018

Published: October 30, 2018

Copyright (C) 2018 by authors and Open Access Library Inc.

This work is licensed under the Creative Commons Attribution International License (CC BY 4.0).

http://creativecommons.org/licenses/by/4.0/

\section{(c) (i) Open Access}

\begin{abstract}
Several years ago, the authors were finally convinced of the reality of the existence of the anomalous neutrino radioisotope (ANRI) absorption. Taking into account the applied and fundamental importance of the effect, as well as the need for thorough repetition and verification of the results of primary research, the authors repeated methodologically and technically the first effective work, but the ANRI-effect registration processes were additionally duplicated using a parallel recording channel operating on another principle (the appearance of a thermal field at decay processes). Both methods: radiometric and thermal, their results coincided both with the data of primary works, and also as well as with each other. Thus, the existence of the ANRI-effect is absolutely reliably proven.
\end{abstract}

\section{Subject Areas}

Particle Physics

\section{Keywords}

Anomalous Neutrino Radioisotope (ANRI) Absorption, Solar Neutrino

Stream, Radiometric and Thermal Methods for Registration Stream

\section{General Remarks}

Five year Jubilee of Discovery Abnormal Neutrino Radio-Isotope (ANRI) absorption were began of existing in time from appear [1] [2] publisher article. Authors very much obliged to Publish House "Natural Science". One of the important tasks of the present work is not only to show again the existence of the anomalous neutrino radioisotope (ANRI) absorption effect, but also to confirm the operability and scientific prospects, simplicity and accessibility of the primary experimental scheme and research methodology [1] [2]. Indeed, in the initial 
studies, the constant interaction of the solar neutrino flux with various physical systems was shown when it was recorded with various measuring instruments [3] [4] [5]. At the same time, the authors, in the interests of their colleagues and involuntarily, carried out work within the framework of the already established paradigm, but the fertile topic allowed a wide variety of studies [1]-[24]. The most relevant of these border studies are also addressed in the overall context of research.

The effectiveness of research has contributed to:

- the absence of a direct prototype and even an analogue,

- lack of even signs of financing;

- the absence in the generally accepted understanding of any experimental base;

- a deep intellectual search based on selected and submitted earlier hypothesis-insights;

- The simplest scheme of the experiment with recording the results, allowing to estimate the existence of the effect in a reliable way and to make a statistical evaluation;

- accessible and reliable repeated testing in any conventional physical laboratory;

- The results should be easily used in other current research and even industry and give tangible results.

In addition to the foregoing "vain" provisions in the conclusion, we try to indicate how best to move on to the more fundamental aspects of the ANRI effect.

\section{Experimental Approaches, a Brief Review of the Work on the Periodicities of Radio Nuclides}

Since neither the characteristics of the Sun, nor the properties of radioactive isotopes for 5 years have changed, we will further adhere to the technique of primary work [1] [2] with the discovery (awareness), as it became obvious after 1 2 years of the ANRI effect. Even then, to increase the reliability, another installation was used, the results of measurements on which completely confirmed the open effect, but were less well known [5]. Therefore, let us briefly report on this new technique and equipment. The determination of the spectra of time variations of radioactivity levels of samples from a mixture of barium 133 with a half-life of 10.54 years and radium 226 with a half-life of 1600 years, weight of $1.3 \mathrm{~kg}$ was carried out according to the following procedure. The sample was placed in a $\gamma$-spectrometer having a resolution of $\sim 0.3 \mathrm{keV}$ and a range from $6.3 \mathrm{keV}$ to 2.63 MeV. (Semiconductor gamma spectrometer Ortec-65195-P/DSPecPlus, No. 41-TP41087A Certificate of state metrological verification No. OR13.21, valid until 21.03.2014, "DOZA"). During 30 seconds there was a signal accumulation, then an amplitude $\gamma$-spectrum of signals was obtained. Then, two spectral harmonic lines were selected for barium in $356 \mathrm{keV}$ and radium in $352 \mathrm{keV}$ and their amplitudes obtained during the accumulation time of $30 \mathrm{sec}$ were deter- 
mined. Then all this was repeated. In this way, the variations in the $\gamma$-radiation of barium and radium were obtained with a time step of $\sim 48 \mathrm{~s}$. and a total duration of more than $5000 \mathrm{~s}$. After receiving these realizations, two other gamma-ray spectral lines of barium and radium were chosen at $161 \mathrm{keV}$ and 186.6 $\mathrm{keV}$ and two more implementations were also made using the same technique as the first ones. Amplitudes of $\gamma$-variations, in contrast to variations of $\beta$-radiation, exceeded 20\% [25]-[33]. Similarly, other records for isotopes of americium and cesium were processed; Spectra of realizations of $\gamma$-variation of Am241-55.5 keV ammonium and Cs137-661 keV cesium are obtained. All the periods of oscillations of the variations in the level of the radioactivity of the isotopes observed on the spectra coincide with the periods of oscillations of the standard model of the sun with the relative content of heavy elements $Z=0.02$ [34].

The gamma-radioactivity of the elements under conditions of dynamic gravity and spin influence was investigated [4]. It was found that irrespective of the physical conditions of the presence of heavy unstable radioactive nuclei, their decay process is modulated by an alternating flux of solar neutrinos. The level of activity of heavy unstable radioactive nuclei as an open system is influenced by three parameters: spin influence (centrifuge rotations), dynamic gravity (centrifugal forces), solar neutrino flux. For certain relations between the indicated parameters, self-organization elements are displayed, which are displayed in the structure of the activity level. Some of these parameters for a radioactive source can be determined experimentally.

Comparing the results, experiments and observations performed on the basis of studies of $\beta$ radioactivity of sources with gamma radiation component [25]-[33] and the results of similar studies, but based on gamma radioactivity [1 \pm 16$]$, we see qualitatively new informatively of gamma radiation from radioactive sources and prospects of application. What is the reason? Let's address to primary representations of all 3 types of radioactivity. At present, the main picture of $\alpha, \beta, \gamma$-radioactivity is well known (Figure 1).

According to Figure 1, during the $\alpha$-decay process, only the upper structures of the nucleus undergo strong changes; we can assume that with $\beta$-decay, the yield of an electron or a positron is also not related to the deep structures of the nucleus; and only when the $\gamma$-decay affects the entire mass of the nucleus, which determines the qualitatively different information content of $\gamma$-decay monitoring [1]-[24]. Moreover, the new properties of the solar neutrino flux and the observed features of their interaction with radioactive substances have led to new studies of other physical systems and processes [6] [7]. Discussions of the features of $\alpha, \beta, \gamma$-radioactivity can try to formalize the ANRI effect by using the idea of V. I. Man'ko and co-authors [35]. In this paper they showed that the interaction efficiency increases with the interaction of an elementary particle with a correlated quantum system. The point is that in this case the Heisenberg ratio changes, which takes the form [35]. 


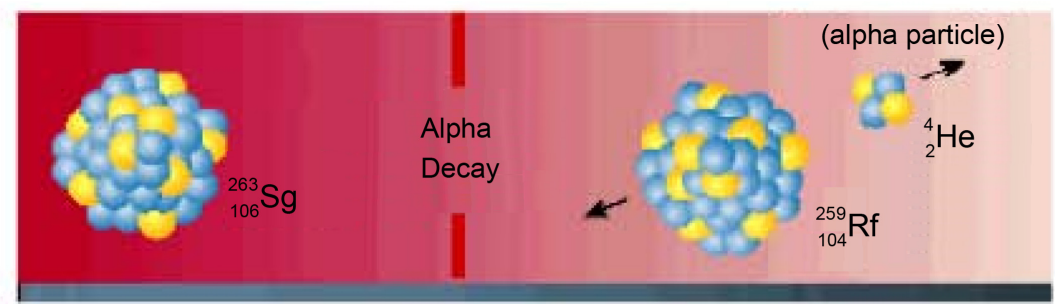

a-radioactivity

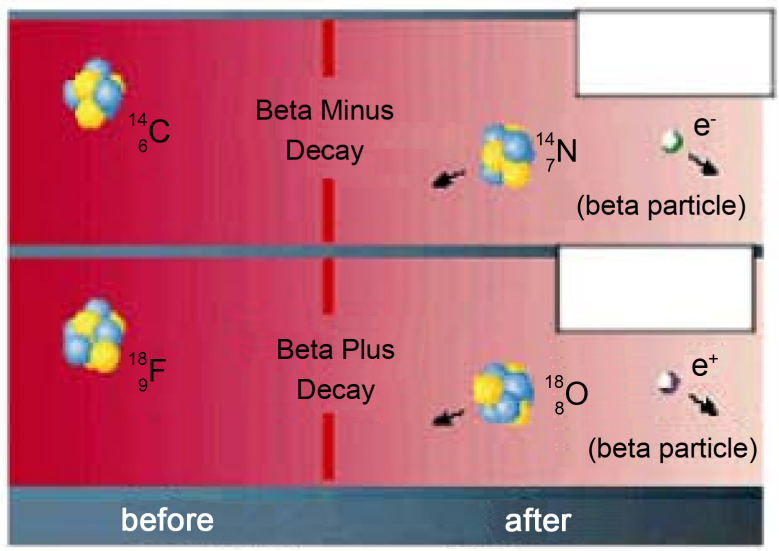

$\beta$-radioactivity

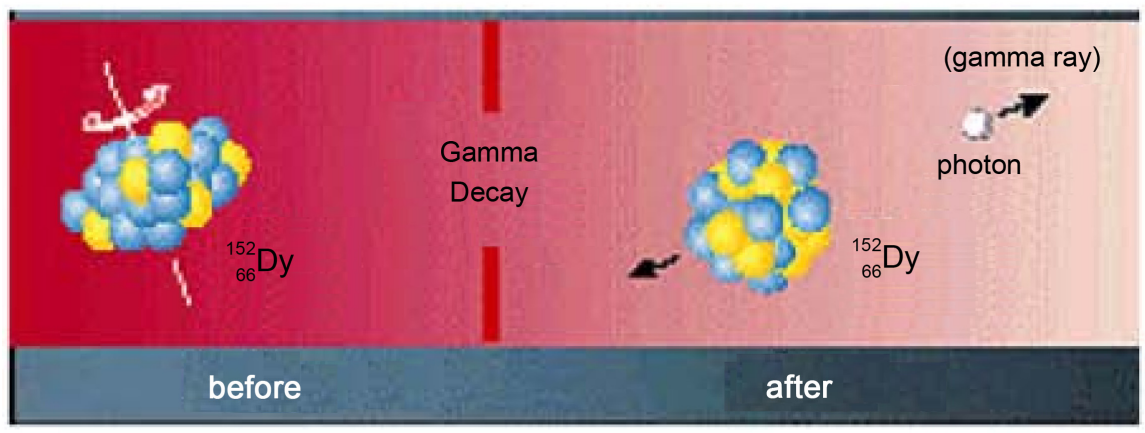

\section{Y-radioactivity}

Figure 1. Illustrative mapping of $\alpha, \beta, \gamma$ - radioactivity's.

$$
x p-p x \geq \frac{\pi h}{1-r},
$$

where, the quantity $r<1$ characterizes the degree of correlation of the quantum system. It is precisely the strong correlation of solar radioactivity (its high degree of structuring with many periods) that we explain the strong impact of solar activity on the cosmic and solar neutrinos. As a special case, this phenomenon can be used to change the half-life of $\alpha, \beta, \gamma$ active radionuclides, nuclear waste. Proceeding from the already existing applied and fundamental importance of the ANRI-effect, it becomes necessary to once again make sure of the inviolability of its experimental basis. 


\section{Repeated Experimental Studies (Similar Observations - 5 Years Later)}

As before, the records of variations in the gamma radiation of the ore were conducted at the facility according to the scheme [1,2] with 3-minute averaging (Figure 2). The in formativeness of recording the variations in the temperature of radioactive sources turned out to be no less interesting records obtained from the radiometer, which made it possible to have one more channel-(temperature), realized on a simpler and more reliable equipment (Figure 3), also confirming the existence of the ANRI effect, in more detail [23].

The main feature of the device in Figure 3 is the complete absorption, including secondary particles. On the other hand, the study of the ANRI effect for a number of problems must be carried out in the field, for a long time (months, years) without changing the hardware blocks, in vibration or in space, and continuously recording one of the main parameters of the radiation field variations or the ANRI effect-heat flow from the decay of radioactive elements.

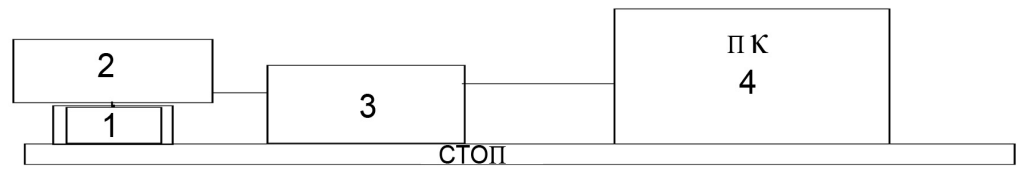

Figure 2. Diagram of registration of gamma-ray variations. 1-Container, 2-radiation detector, 3-digital recorder, 4-personal computer.

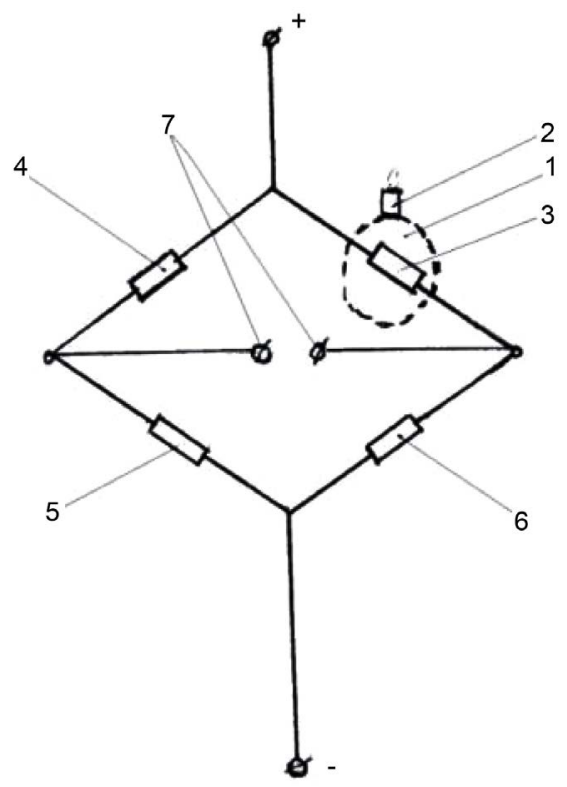

Figure 3. Principal (bridge) scheme of the radiometer on heat flows (RHF): 1-radioisotope mass; 2-classical radiometer; 3-calibrated thermocouple; 4 - 6-the resistors calibrated with respect to the temperature-sensor resistor 3; 7-output of a useful signal. 
Externally, the nature of the signal recording on the radiometer (Figure 2) did not change. As well as earlier received records it was necessary to compare with the existing state of the Sun. Since initially it is difficult to single out the criterion of the current state of the Sun, preference was again given to the $\mathrm{K}_{\mathrm{p}}$ index as the most general, reflecting both the activity of the Sun and the processes in deep space. The definition of the $\mathrm{K}_{\mathrm{p}}$-index ${ }^{*}$ is more formal and limited. During the same time interval of gamma radiation observation, a series of $\mathrm{K}_{\mathrm{p}}$ index was constructed (Figure 3). To compare the parallel records of the gamma-ray series and the $\mathrm{K}_{\mathrm{p}}$-index, synchronous variations in the gamma radiation of the ore were averaged over 3-hour intervals. Then, the sliding correlation function was determined in a 60-hour window (20 independent points). There is from 10.02.2018 to record solar $\mathrm{K}_{\mathrm{R}}$ indices (Figure 4).

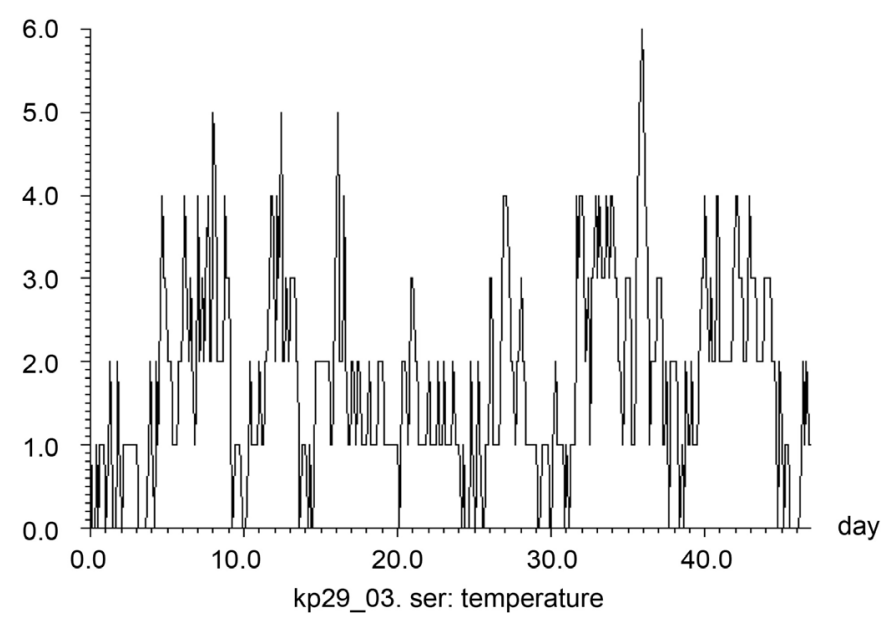

Figure 4. Pre-processed $\mathrm{K}_{\mathrm{p}}$ index record.

After 3 - 4 days, the recording of variations in the radioactivity of uranium ore and variations in the temperature of uranium ore began with a three-hour averaging (the activity level is 1 day earlier than the temperature), Figure 5 and Figure 6. The in formativeness of recording the variations in the temperature of

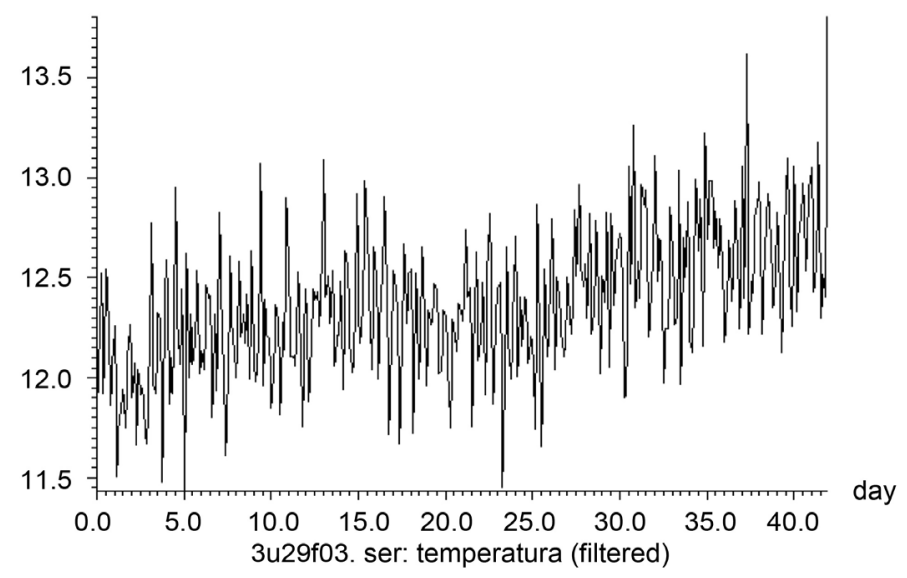

Figure 5. Recording variations in a level of the activity of uranium ore. 


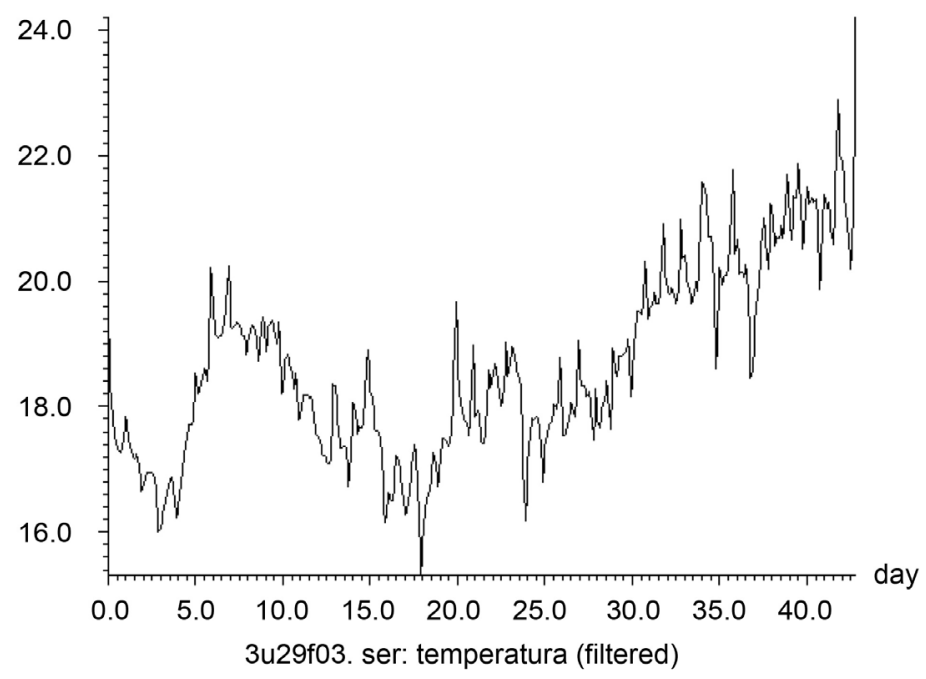

Figure 6. Recording variations in the temperature level of uranium ore.

radioactive sources turned out to be no less interesting records obtained from the radiometer, which made it possible to have one more channel (temperature), implemented on more simpler and reliable equipment, in more detail [23].

$\mathrm{K}_{\mathrm{p}}$-index is global planetary index of geomagnetic activity. The $\mathrm{K}$-index is a three-hour quasi-logarithmic local index of geomagnetic activity relative to the calm day curve for a given location. The $\mathrm{K}_{\mathrm{p}}$-index measures the deviation of the most disturbed horizontal component of the magnetic field at stationary stations around the world from their own local $\mathrm{K}$-indices. Then the global $\mathrm{K}_{\mathrm{p}}$-index is determined by an algorithm that combines the average values of each station. The $\mathrm{K}_{\mathrm{p}}$-index ranges from 0 to 9 , where the value 0 means no geomagnetic activity, and the value 9 means an extreme geomagnetic storm.

In addition to the variations in temperature, the ores were obtained during the same time interval and the temperature variations Cs137, Figure 7.

Further records were processed. The obtained data went through a filtration, excluding the daily component of periodicity, in spite of the fact that some of the investigated processes have this component. Then, the functions of cross correlation (FCC) between the series of processed registration data and data on $\mathrm{K}_{\mathrm{p}}$ indices, as well as between the rows of primary data, were determined, Figure 8 and Figure 9.

Note the significant $(K=-0.45)$, but negative correlation with delay for 10 days.

In Figure 8 and Figure 9-FCC are similar, and the correlations are significant $(\mathrm{K}=-0.45)$, which also indicates the similarity of the primary processes under study, and the processes that generate them. Next, consider the FCC for recorded other temperature processes and activity (Figures 10-12).

The fact that apart from the main peak associated with the rotation of the Sun (28 - 30 days), there is also a peak related to the 4 th harmonic from the period of rotation, that is, about 7 days, to the features of the spectra (Figure 13 and Figure 14). There is also a complete similarity of the spectra of the FCC. 


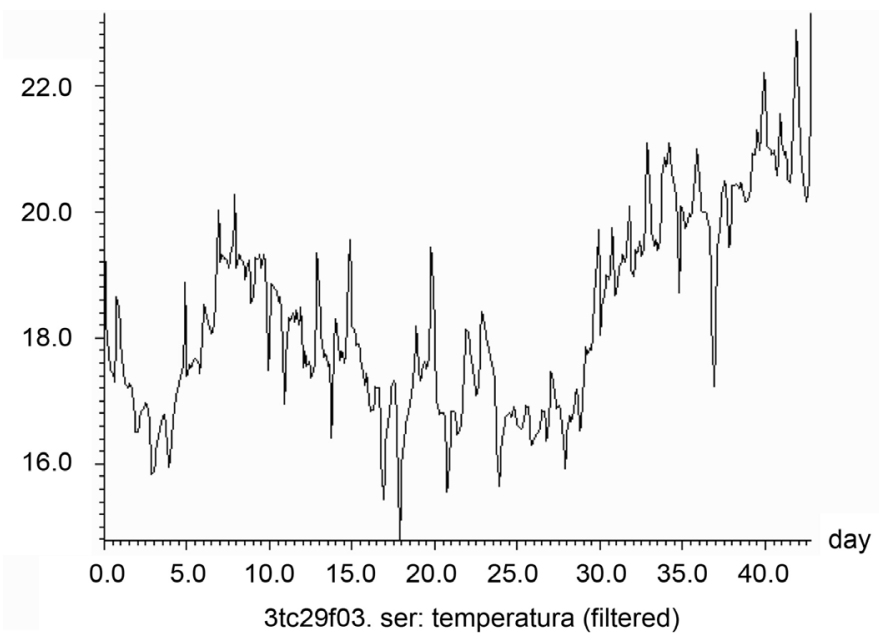

Figure 7. Recording of variations in the temperature level Cs137.

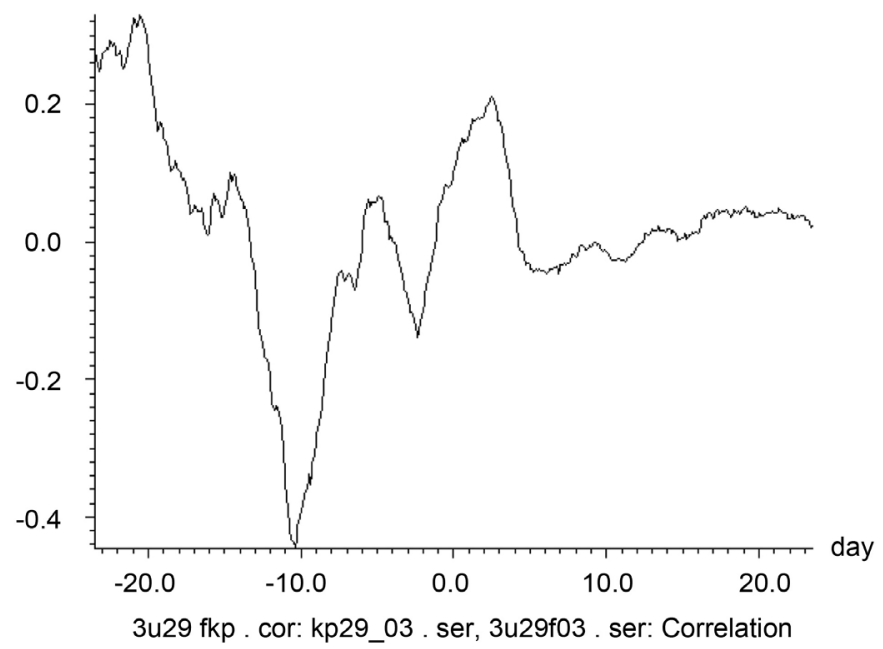

Figure 8. FCC between $\mathrm{K}_{\mathrm{p}}$-indices (Figure 3 ) and variations in radioactivity of ore (Figure 4).

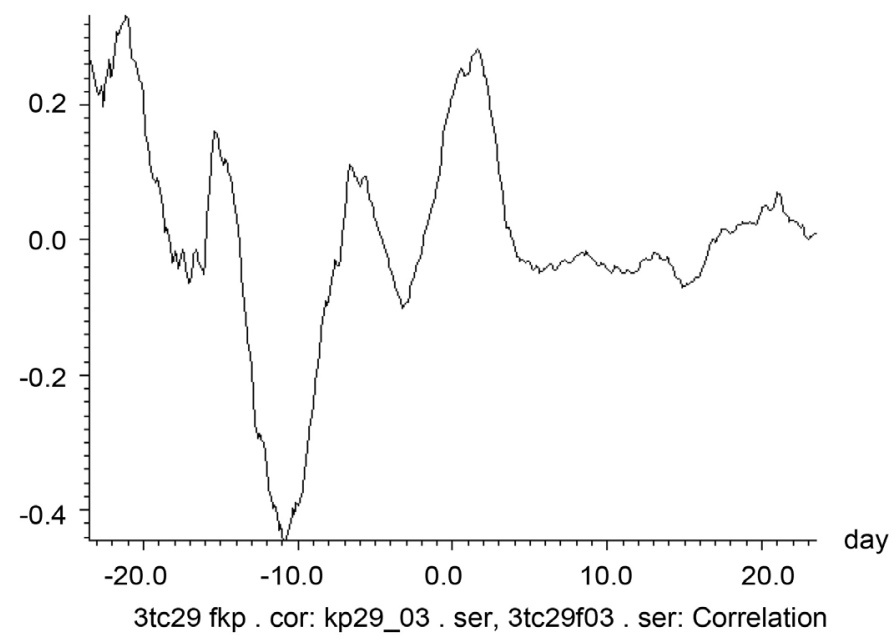

Figure 9. FCC between the series of $\mathrm{K}_{\mathrm{p}}$-indices and the temperature variations Cs137. 


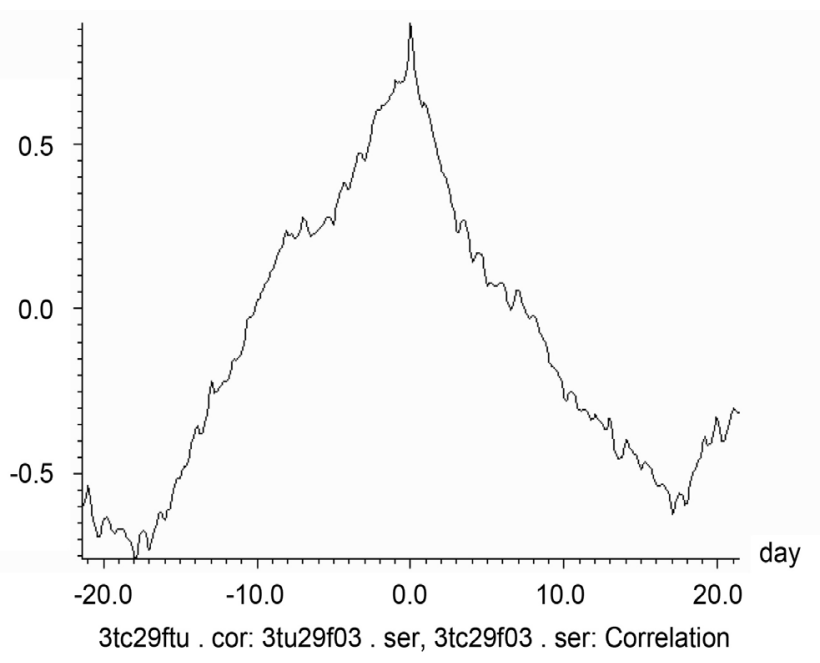

Figure 10. FCC between the records of the temperature variations of Cs137 and the temperature of the ore.

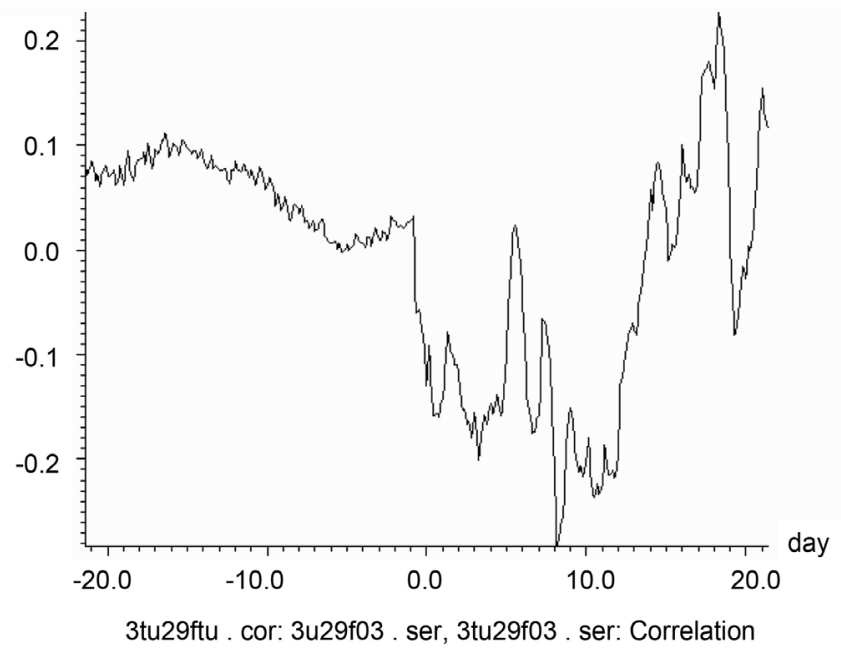

Figure 11. FCC between variations in ore temperature and variations in the radioactivity of the ore.

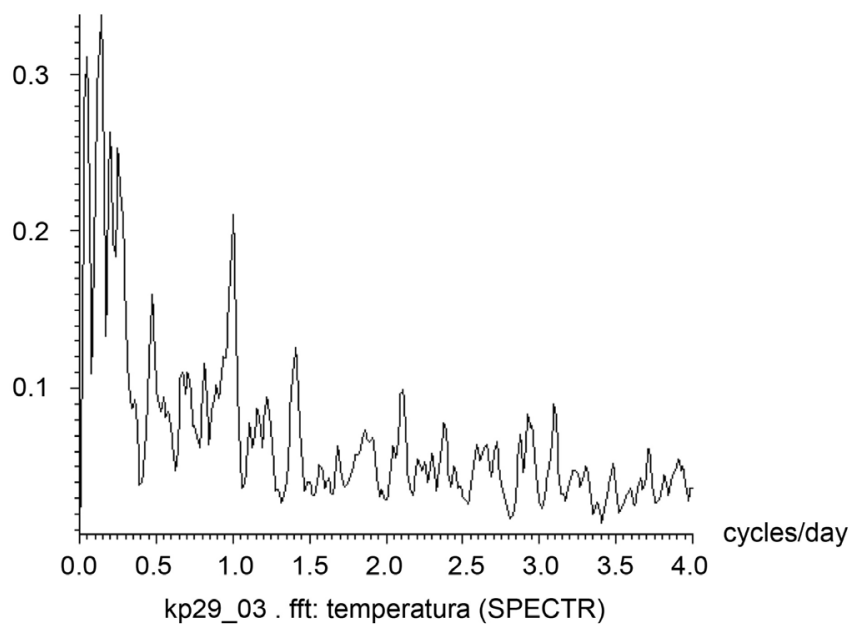

Figure 12. The spectrum of $\mathrm{K}_{\mathrm{p}}$ indices for 1.5 months. 


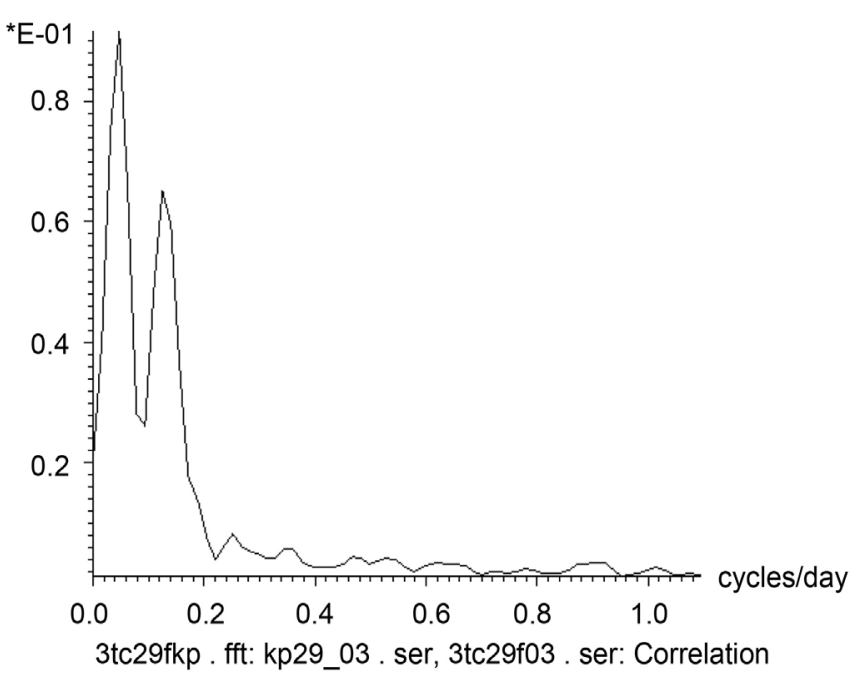

Figure 13. Spectrum of FCC between variations of Kp indices and temperature variations Cs137.

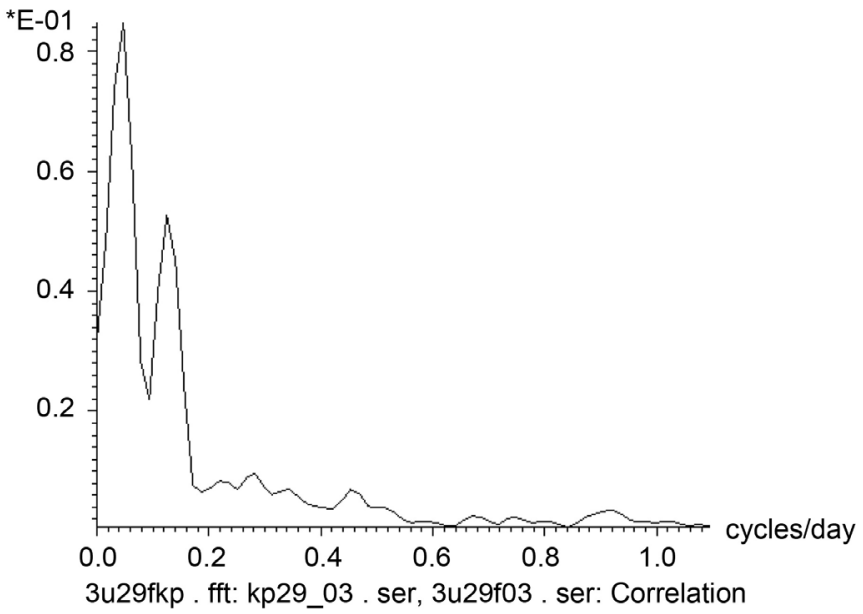

Figure 14. The spectrum of FCC between the variations of $\mathrm{K}_{\mathrm{p}}$ indices and variations in the radioactivity of uranium ore.

In further investigation, the functions of sliding correlation between $\mathrm{K}_{\mathrm{p}}$ indices and variations were determined: the intensity of ore activity (Figure 15); temperature of the ore (Figure 16) and cesium temperature137 (Figure 17).

All graphical representations of the processes are in Figures 15-17.

Graphical representations of the processes of Figures 15-17 are all significant, that is, with a probability $\mathrm{P}>0.95$, in their maximum amplitudes, they exceed a random coincidence. And they are similar to each other.

But the most important conclusion is that complete agreement with the materials and conclusions of primary publications is observed even more widely and diversely above the observational materials on $\mathrm{K}_{\mathrm{p}}$ indices and data on the gamma radiation and temperature of radioactive sources and their subsequent processing $[1,2]$. This allows us to move on to the next stage of the research-searching for a modulation of the activity level of radiation sources at 


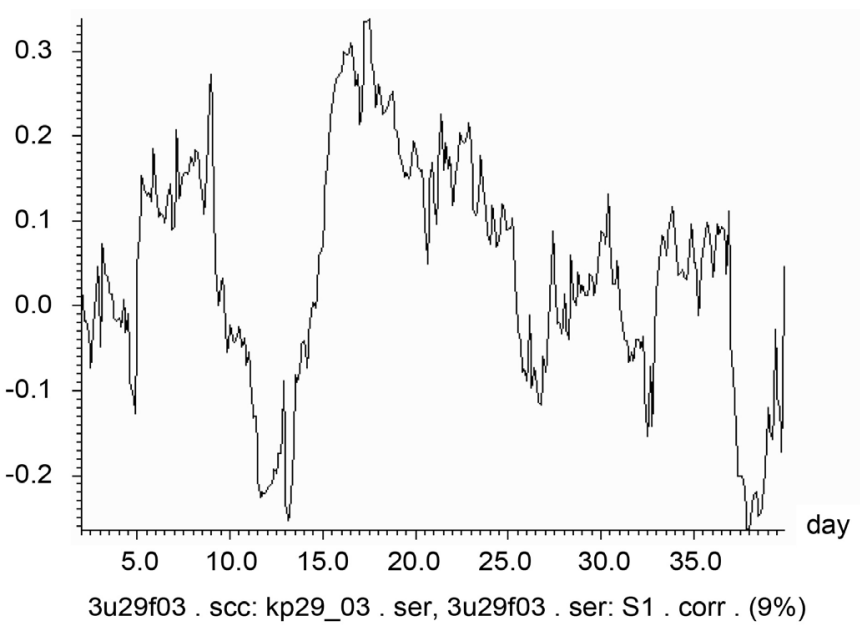

Figure 15. The function of sliding correlation between $\mathrm{K}_{\mathrm{p}}$ indices and variations in the intensity of ore activity.

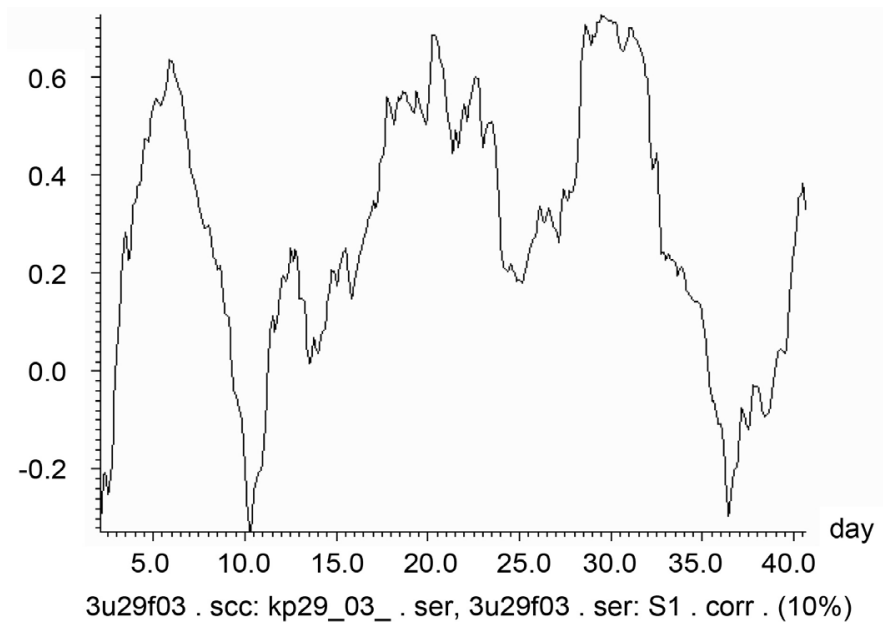

Figure 16. The function of sliding correlation (FSC) between $\mathrm{K}_{\mathrm{p}}$ indices and ore temperature.

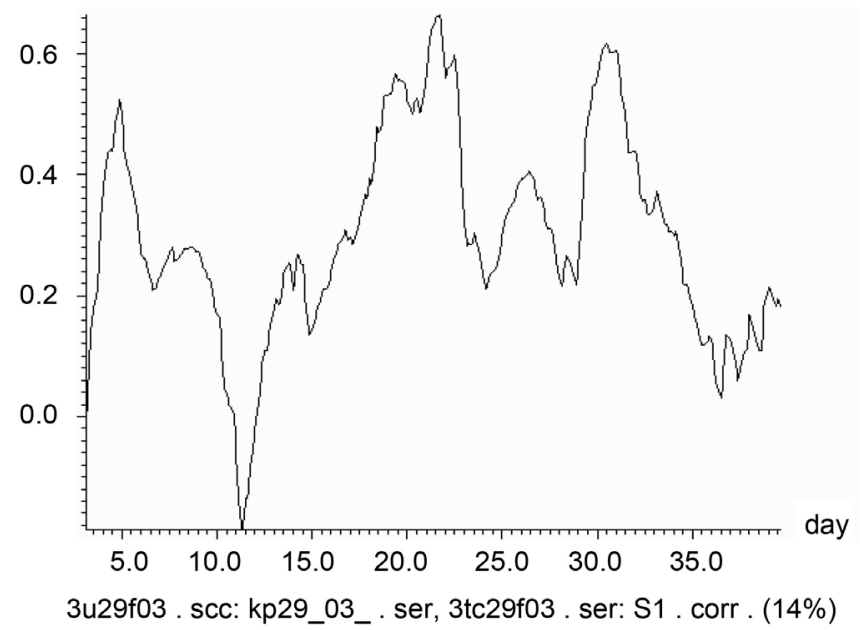

Figure 17. Function of sliding correlation between $\mathrm{K}_{\mathrm{p}}$ indices and variations in cesium 137 temperature. 
periods of the Sun's own oscillations, that is, the final proof of the existence of the ANRI-effect.

For this realization of variations in the intensity of radioactivity, the ores were divided into two sections, in processing of which the energy spectrum was obtained by determining the cross-correlation function and the subsequent Fourier transform, Figure 18, the significant periods of which served as the material for the construction of Table 1 .

In Table 1: columns 2, 3 are the observed significant spectral peaks of the spectrum (Figure 17), hour, min.; column 4-natural oscillations of the Sun (OSO), min [24]; column 5-the OSO modes.

Next, a $10 \%$ sliding-correlation function (FSC) was determined between the spectra of the initial and tail sections of the realization of temporal variations in the intensity of radioactive ore.

The resulting function is positive and significant (Figure 19).

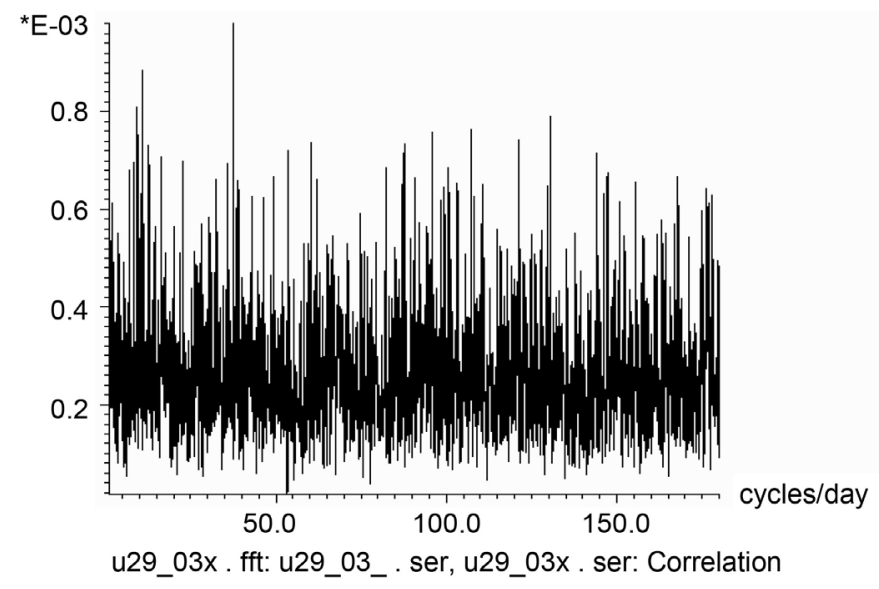

Figure 18. Energetic spectrum of temporary variations calculated for the intensity radioactive ore.

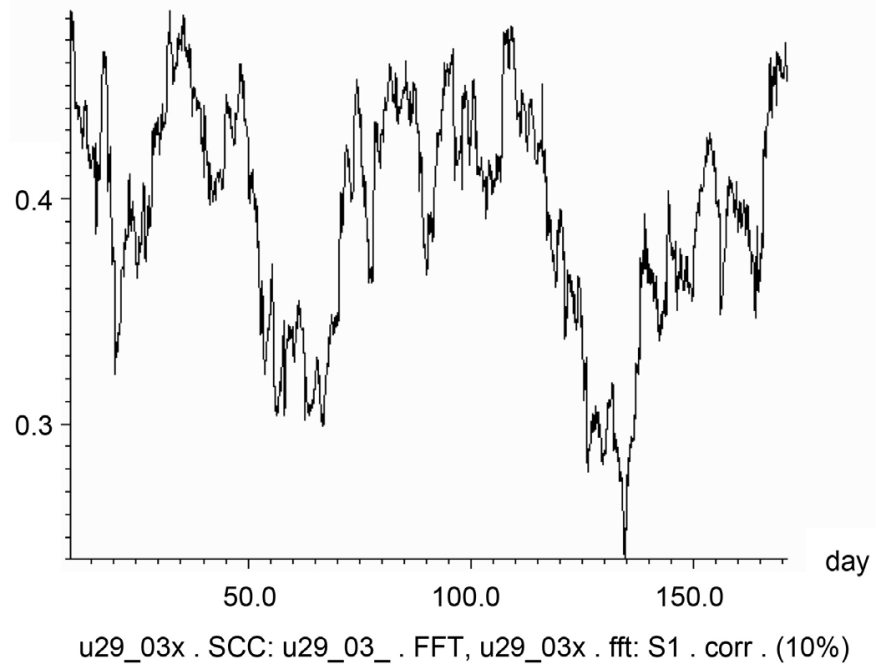

Figure 19. FSC between the spectra's of the initial part and tail of the realization. 
Table 1. The observed of significant periods of the spectrum (Figure 18).

\begin{tabular}{|c|c|c|c|c|c|}
\hline №/№ & $\begin{array}{l}\text { The observed } \\
\text { periods (hour) }\end{array}$ & $\begin{array}{l}\text { The observed } \\
\text { periods (min) }\end{array}$ & $\begin{array}{c}\text { Periods own solar } \\
\text { oscillation (OSO) (min) }\end{array}$ & $\begin{array}{l}\text { Mode } \\
\text { OSO }\end{array}$ & Note \\
\hline 1 & 24 & & & & \\
\hline 2 & 12 & & & & \\
\hline 3 & 3.46 & & & & \\
\hline 4 & 2.94 & 176.2 & 175.9 & g16, 13 & \\
\hline 5 & & 154.6 & 156.5 & $\mathrm{~g} 14,13$ & \\
\hline 6 & & 130.6 & 132.9 & $\mathrm{~g} 8,12$ & \\
\hline 7 & & 112.6 & 111.7 & $\mathrm{~g} 12,11$ & \\
\hline 8 & & 88.5 & 84.4 & g2, 11 & \\
\hline 9 & & 87.1 & 84.4 & g9, 14 & \\
\hline 10 & & 71.8 & 72.6 & g3, 12 & \\
\hline 11 & & 63.4 & 63.01 & $\mathrm{~g} 2,12$ & \\
\hline 12 & & 51.3 & 49.6 & g2, 14 & \\
\hline 13 & & 47.2 & 47.9 & $\mathrm{~g} 1,13$ & \\
\hline 14 & & 44.6 & 44.2 & $\mathrm{~g} 1,14$ & \\
\hline 15 & & 40.1 & 40.9 & $\mathrm{p} 2,10$ & \\
\hline 16 & & 38.4 & 37.6 & $\mathrm{p} 1,14$ & \\
\hline 17 & & 36.94 & 36.98 & $\mathrm{p} 2,11$ & \\
\hline 18 & & 33.0 & 32.19 & $\mathrm{p} 2,12$ & \\
\hline 19 & & 30.8 & 30.93 & $\mathrm{p} 3,10$ & \\
\hline 20 & & 29.2 & 29.42 & $\mathrm{p} 2,13$ & \\
\hline 21 & & 26.7 & 25.1 & $\mathrm{p} 3,12$ & \\
\hline 22 & & 23.8 & 23.21 & p3, 13 & \\
\hline 23 & & 23.16 & 23.21 & $\mathrm{p} 3,13$ & \\
\hline 24 & & 19.27 & 19.26 & $\mathrm{p} 4,13$ & \\
\hline 25 & & 17.45 & 17.39 & $\mathrm{p} 5,12$ & \\
\hline 26 & & 16.48 & 16.44 & $\mathrm{p} 5,13$ & \\
\hline 27 & & 16.36 & 16.44 & $\mathrm{p} 5,13$ & \\
\hline 28 & & 15.91 & 16.04 & $\mathrm{p} 6,11$ & \\
\hline 29 & & 14.98 & 14.93 & $\mathrm{p} 7,10$ & \\
\hline 30 & & 14.3 & 14.08 & $\mathrm{p} 7,11$ & \\
\hline 31 & & 13.96 & 13.81 & $\mathrm{p} 6,14$ & \\
\hline 32 & & 13.43 & 13.35 & $\mathrm{p} 7,12$ & \\
\hline 33 & & 13.03 & 13.21 & $\mathrm{p} 8,10$ & \\
\hline 34 & & 11.88 & 11.86 & $\mathrm{p} 9,10$ & \\
\hline 35 & & 11.12 & 11.14 & $\mathrm{p} 8,14$ & \\
\hline 36 & & 11.1 & 11.14 & $\mathrm{p} 8,14$ & \\
\hline 37 & & 9.99 & 9.97 & $\mathrm{p} 10,12$ & \\
\hline 38 & & 9.86 & 9.90 & $\mathrm{p} 11,10$ & \\
\hline 39 & & 9.79 & 9.65 & $\mathrm{p} 10,13$ & \\
\hline 40 & & 9.77 & 9.65 & $\mathrm{p} 10,13$ & \\
\hline 41 & & 9.55 & 9.54 & $\mathrm{p} 11,11$ & \\
\hline 42 & & 9.25 & 9.21 & $\mathrm{p} 11,12$ & \\
\hline 43 & & 8.89 & 8.84 & $\mathrm{p} 12,11$ & \\
\hline 44 & & 8.82 & 8.84 & $\mathrm{p} 12,11$ & \\
\hline 45 & & 8.57 & 8.56 & $\mathrm{p} 12,12$ & \\
\hline 46 & & 8.42 & 8.50 & $\mathrm{p} 13,10$ & \\
\hline 47 & & 8.24 & 8.23 & $\mathrm{p} 13,11$ & \\
\hline
\end{tabular}


FSC Figure 20 almost everywhere is at the level of unity, which indicates a practical coincidence of the spectra and the effectiveness of the new temperature method.

Thus, after 5 years, an even more numerous set of proper periods of the solar oscillations was obtained again in full agreement with the data $[1,2]$, and also [4, 5] using new sources of gamma radiation and additionally using a new independent (thermal) recording channel. Note, after a second, even deeper proof of the existence of the ANRI-the effect first discovered in the studies of 2013-2015, further understanding of the ANRI effect was further studied and developed in [6] [7] [9]-[14] [18] [19] [21] [23] [24] [25]. An important role in this development was played by the experimental estimate of the capture cross section for neutrinos during its interaction with radioactive isotopes [12] [20]. This value was $\sim 10^{-12}$, which is 30 orders of magnitude $\left(10^{30}\right)$ higher than the generally accepted one for the case of neutrino interaction with a passive medium. For completeness and breadth of representation of the state of this problem, we turn to borderline and/or similar studies and publications. Perhaps the first comparative analysis was given in [8], where on the basis of a brief survey it was noted.

The existence of periodicity or simply perturbations in the level of radioactivity of various sources has been discovered quite a long time [25]-[33]. Thus, the temporal variations of the radioactive decay (decay numbers) of Cs-137 were registered in April 19-23, 1994, (Baurov Yu. A.) and other researchers. Temporal variations in the analysis of records of beta radioactivity have long periods, while short variations are practically not distinguished, but in duration they are limited to several days or more. The annual variation of the Sun-Earth distance is well-marked. But only variations in the analysis of gamma radioactivity records, as was shown above, have a wide range of periods (from a few minutes to a day or more). All their spectra contain reliable periods of intrinsic solar oscillations

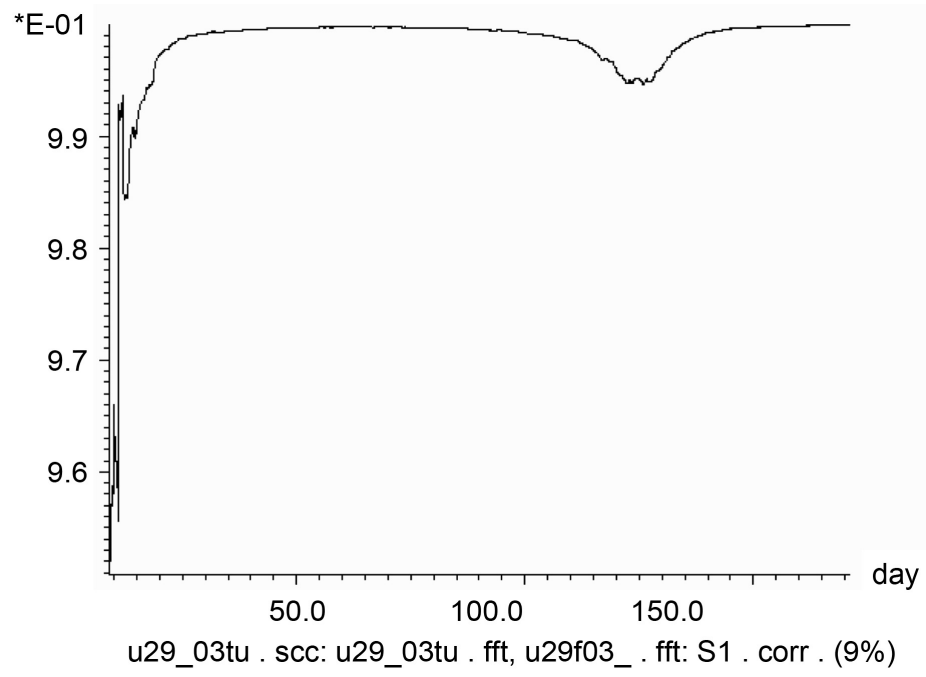

Figure 20. FSC in a $9 \%$ window between the spectrum of ore temperature variations with the filtered daily component and the spectrum of variations in radioactivity of the ore. 
(up to 40 periods), which is observed only in [1] [2] [4] [5]. In this case, long periods of solar oscillations are found in seismic oscillations of the Earth and the Moon in the analysis of wave fields as a result of the interaction of a modulated flux of solar neutrinos with radioactive rocks of these celestial bodies [9] [11] [14] [16]. That is, the effects of neutrino flux interaction with gamma radiation sources (ANRI effect) will allow deeper exploration of the Sun, seismicity of the Earth and other applied research. Periodicity studies are most effective in monitoring $\gamma$-radioactivity.

In this paper, we analyzed border foreign publications (the exception is Parkhomov), relating to the past primary stage 3 to 8 years before the discovery of the ANRI effect. But this does not mean that at the present time there has come a "lull", there is a lively discussion about the physical mechanism of the long-period and even non periodic influence of the Sun primarily on the beta-radioactive decay of nuclei. The mechanism of influence through changes in the structure of space is discussed, and one of the groups on the unique apparatus showed the absence of this mechanism.

In recent years, in addition to Parkhomov in Russia appeared publications of a group of professionals who are directly involved in the study of neutrinos. But the essence of their rather recent publications boils down to a repetition of foreign works 10 to 15 years ago. As for the latest foreign publications, the paper [36] is of special interest, where the decay of radon gas was studied on the basis of hourly gamma measurements. Particular attention is paid to fluctuations with frequencies of 11.35 years and 12.63 years, similar to those of the Brookhaven National Laboratory (Sturrock et al., 2016). The frequency of 12.63 years corresponds to the synodic rotation frequency of the radiation zone determined by helioseismology. 11.35 g. - corresponds to the half-life of the Sun, and corresponds to the influence of neutrinos. It is important that the researchers could not identify the influence of the environment or the experimental artifact or any combination thereof as interference. It is quite obvious that if the authors [36] switch to our methodology and analogous equipment, much of the physics of the Sun will become more accessible.

\section{The Conclusion}

Five years of experience in the experimental development of studies of various forms and systems for recording the interaction of neutrinos with radioactive materials by monitoring the level of radiation and/or heat flux (later work) has proved the existence of the ANRI effect. Targeted experiments have determined a new capture cross section for neutrinos under the ANRI effect, the applicability and/or manifestation of which is observed in many processes and phenomena in physics and geophysics.

\section{References}

[1] Khavroshkin, O.B. and Tsyplakov, V.V. (2013) Radioactivity of a Sample of Ore: 
Monitoring. Engineering Physics, No. 8, 53-62.

[2] Khavroshkin, O. and Tsyplakov, V. (2013) Sun, Earth, Radioactive Ore: Common Periodicity. The Natural Science (NS), 5, 1001-1005.

[3] Khavroshkin, O. and Tsyplakov, V. (2011) Radioactivity of the Nuclei in a Centrifugal Force Field. The Natural Science (NS), 3, 733-737.

[4] Khavroshkin, O.B. and Tsyplakov, V.V. (2013) Natural Radioactivity as an Open System. Engineering Physics, No. 12.

[5] Starodubov, A.V., Khavroshkin, O.B. and Tsyplakov, V.V. (2014) From Periodicity of Radioactivity to Cosmic and Metaphysical Oscillations. Metaphysics, No. 1, 137-149.

[6] Khavroshkin, O.B. and Tsyplakov, V.V. (2014) Hydrogen Maser: Solar Periodicity. Engineering Physics, No. 3, 25-31.

[7] Khavroshkin, O.B. and Tsyplakov, V.V. (2014) Atomic-Molecular Metastable Media and Solar Neutrinos. Engineering Physics, No. 6, 40-47.

[8] Rukhadze, A.A., Khavroshkin, O.B. and Tsyplakov, V.V. (2015) Periodicity of Natural Radioactivity. Engineering Physics, No. 6, 26-36.

[9] Khavroshkin, O.B. and Tsyplakov, V.V. (2015) Modulated Neutrino Fluxes: Astrophysical and Geophysical Periodicity. Engineering Physics, No. 10, 27-47.

[10] Khavroshkin, O. and Tsyplakov, V. (2016) The Radioactivity of Nuclei \& Solar Oscillations: New Experiments. NS, 8, 20-32.

[11] Tarasov, N.T., Tarasova, N.V., Khavroshkin, O.B. and Tsyplakov, V.V. (2016) Flash of the Top SN1987A: Seismic Response. Engineering Physics, No. 6, 82-93.

[12] Karagioz, O.V., Izmailov, V.P., Khavroshkin, O.B. and Tsyplakov, V.V. (2016) Kavvendish's Terrestrial Weights and Cern's Hadron Collaider: Different Destinies and Results. Engineering Physics, No. 4, 3-8.

[13] Khavroshkin, O.B. and Tsyplakov, V.V. (2016) Anomalous Neutrino Radioisotopic (ANRI) Absorption and Nuclear Reactor. Engineering Physics, No. 10, 3-8.

[14] Khavroshkin, O.B. and Tsyplakov, V.V. (2017) Solar Neutrino, Monopol, Dion, Axion: Search Experiments. Applied Physics and Mathematics, No. 2, 3-10.

[15] Khavroshkin, O.B. and Tsyplakov, V.V. (2016) Gravitational Wave Experiment: Geophysical and Astrophysical Components. Engineering Physics, No. 8, 56-63.

[16] Nikolayev, A.V., Khavroshkin, O.B. and Tsyplakov, V.V. (2016) Structure of Astrophysical Components of Seismic Emissions and Noise. Engineering Physics, 9, 69-73.

[17] Khavroshkin, O.B. and Tsyplakov, V.V. (2018) Devices for Advanced Physics Research. Invention Journal of Research Technology in Engineering \& Management, 2, 59-63.

[18] Khavroshkin, O.B. and Tsyplakov, V.V. (2017) Neutrinos: Experiments, New Results. Palmarium Academic Publishing, 265 p.

[19] Khavroshkin, O.B. and Tsyplakov, V.V. (2017) Anomalous Neutrino Radioisotope (ANRI) Absorption and Background Antineutrinos of a Water-Water Nuclear Reactor. SciFed Journal of Nuclear Science, 1, 1.

[20] Khavroshkin, O.B. and Tsyplakov, V.V. (2017) Cavendish Torsion Balance and Hadron Collider at the Cern: Different Fates and Results. SciFed Journal of Nuclear Science.

[21] Khavroshkin, O.B. and Tsyplakov, V.V. (2017) Yellowstone's Volcano and Sun: Fragments Selected Works. Journal of Geology and Geoscience, 1, 1-5. 
[22] Rukhadze, A.A., Khavroshkin, O.B. and Tsyplakov, V.V. (2017) Graviton and Neutrino: Modulated Streams or Waves of the Envelope. Engineering Physics, No. 10, 71-85.

[23] Khavroshkin, O.B. and Tsyplakov, V.V. (2017) Temperature Fields of Radioactive Substances: Time Variations and Applications. Engineering Physics, No. 7, 17-32.

[24] Khavroshkin, O.B. and Tsyplakov, V.V. (2018) Devices for Advanced Physics Research. Invention Journal of Research Technology in Engineering \& Management, 2, 59-63.

[25] Baurov, Y.A., Sobolev, Y.G. and Ryabov, Y.V. (2014) New Force, Global Anisotropy and the Changes in b-Decay Rate of Radioactive Elements. American Journal of Astronomy and Astrophysics, 2, 8-19.

[26] Baurov, Yu.A. (2000) On the Structure of the Physical Vacuum and a New Interaction in Nature (Theory, Experiment and Applications). Nova Science, New York.

[27] Parhomov, A.G. (2014) Periodic and Sporadic Changes in the Rate of Beta Decays, Observed during Long-Term Observations. Metaphysics, 1, 124-136.

[28] Jenkins, J.H., et al. (2012) Additional Experimental Evidence for a Solar Influence on Nuclear Decay Rates.

[29] Lobashov, V.M. (2003) Measurement of the Mass of Neutrinos in Beta-Decay of Tritium. Vestnik, 73, 14-27.

[30] Baurov, Yu.A., Sobolev, Yu.G., Kushniruk, V.F., et al. (2000) Experimental Investigations of Changes in Velocity in the Rate of Beta Decay of Radioactive Elements. Physical Physics of Russia, No. 1, 1-7.

[31] Baurov, Y.A., Konradov, A.A., Kushniruk, V.F., et al. (2001) Experimental Investigation of Changes in the Beta-Decay Rate of ${ }^{60} \mathrm{Co}$ and ${ }^{137} \mathrm{Cs}$. Modern Physics Letters $A, 16,2081-2101$.

[32] Falkenberg, E.D. (2001) Radioactive Decay Caused by Neutrinos? Aperion, 8, 2081-2101.

[33] Fischbach, E., Buncher, J.B., Gruenwald, J.T., et al. (2009) Time Dependent Nuclear Decay Parameters: New Evidence for New Forces? Space Science Reviews, 145, 285-335.

[34] Iben, I. Jr. and Mahaffy, J. (1976) On the Sun's Acoustical Spectrum. Astrophysical Journal, 209, L39-L43. https://doi.org/10.1086/182262

[35] Dodonov, V.V., Klimov, A.B. and Man'ko, V.L. (1996) Low Energy Wave Packet Tunneling from a Parabolic Potential Well through a High Potential Barrier. Physics Letters A, 41-48.

[36] Sturrock, P.A., Steinitz, G. and Fiscbach, E. (2018) Analysis of Gamma Radiation from a Radon Source. II: Indications of Influences of Both Solar and Cosmic Neutrinos on Beta Decays. Astroparticle Physics, 100, 1-12. https://doi.org/10.1016/j.astropartphys.2018.02.003 